\title{
Dynamics of plasma expansion in the pulsed laser material interaction
}

\author{
N KUMAR*, S DASH, A K TYAGI and BALDEV RAJ \\ Surface and Nanoscience Division, Indira Gandhi Centre for Atomic Research, \\ Kalpakkam 603102 \\ e-mail: niranjan@igcar.gov.in
}

MS received 10 July 2009; revised 3 January 2010; accepted 20 January 2010

\begin{abstract}
A pulse Nd: YAG laser with pulse duration 5-10 ns, beam radius at focal point $0 \cdot 2-0.4 \mathrm{~mm}$, wavelengths $1064 \mathrm{~nm}, 532 \mathrm{~nm}$ and $238 \mathrm{~nm}$ with linearly polarized radiation and Gaussian beam profile, was impacted on a thin foil of titanium metal for generating plasma plume. Numerically, the above parameters were linked with average kinetic energy of the electrons and ions in the laser-induced plasma. In the present model, electrons having higher velocities are assumed to escape from plasma, that forms a negatively charged sheath around the plasma. It is seen from present computations that the forward directed nature of the laser evaporation process results from the anisotropic expansion velocities associated with different species. These velocities are mainly controlled by the initial dimension of the expanding plasma. An attempt was undertaken to estimate the length of the plume at different ambient gas pressures using an adiabatic expansion model. The rate of the plasma expansion for various $\mathrm{Ar}^{+}$ion energies was derived from numerical calculations. A numerical definition of this plasma includes events like collisional/radiative, excitation/de-excitation and ionization/recombination processes involving multiples of energy levels with several ionization stages. Finally, based on a kinetic model, the plasma expansion rate across the laser beam axis was investigated.
\end{abstract}

Keywords. Pulsed laser; plasma expansion; plasma ionization; plume dimension.

\section{Introduction}

A thin solid titanium (Ti) target was mounted inside a vacuum chamber of a pulsed laser deposition (PLD) facility. Typical surface atom density of this Ti specimen is $\sim 10^{16}$ atoms $/ \mathrm{cm}^{2}$. This was heated by a pulsed-laser radiation to very high temperature. The process of plasma generation from the solid Ti target in initial stages is quite complex. It is necessary to consider ballistic heating of the target in the condensed phase which leads to melting, vaporization and ionization. High irradiance causes ultra fast ionization. The energy required to ionize

*For correspondence 
an atom is rather negligibly small compared to the energy costs for heating a target to some millions of Kelvin. On this basis, in many calculations, the initial stages of the plasma production from solid target are ignored and fully ionized cold plasma is assumed to exist where ion and electron densities roughly equal the solid atom density. Results reported by Anisimov et al (1974), Mazhukin et al (2002) and Noll et al (2004) may be referred. The dynamics of plasma formed from solid target are governed by the geometry of the skin layer of the target and the profile of the incident laser beam in accordance with the results reported by Colonna et al (2001) and Furusawa et al (2004).

One of the most distinguishing features of plasma interaction with Ti metal is associated with ionization of the proximal gas entraining flow of $\mathrm{Ti}$ atoms, ions and free electrons as reported by Itina et al (2003). As a result, charged particles get accumulated in plasma. Thus, a local electrostatic field is set-up which influences the motion of electrons and ions. This phenomenon is reported by Casavola et al (2003); Belotserkovsky \& Davidov (1982) and Giacomo et al (2001). In general, charge, momentum and energy flow depend on spatial electrostatic potential distribution around particles. Kinetic description of plasma-particle interaction involves a combined solution of the Boltzmann's equation for the velocity distribution functions of molecules, electrons and ions on one hand and the Poisson equation for the electrostatic potential on the other.

The experimental data, however, suggests that the maximum energies of the ions are independent of variation in their charge states as reported by Gornushkin et al (2001) and Ho et al (1996). However, if accelerating fields are responsible for the high ion velocity, one would expect that ions in the highest charge state should have a relatively higher maximum energy than those in the lower charge states. Moreover, the observation that neutral molecules are emitted with kinetic energies of the some order of magnitude as the ions, contradicts this postulate. Most of the experimental evidence indicates that the high-density plasma that are created in the laser-surface interaction, expands as predominantly neutral plasmas with the ions and electrons travelling together.

The interpretations of most of the experimental results seem to be inconsistent with the hypothesis that electrons separate from the ions in considerable quantity and are accelerated by electrostatic interaction reported by Knight (1979); Mazhukin et al (2002) and Rethfeld et al (2004).

In the present paper, computations carried out to analyse nature of the laser evaporation process, results from the anisotropic expansion velocities associated with different plume species. The rate of the plasma expansion for various ions energies have been derived from the numerical calculations. Using a kinetic model, the plasma expansion rate across the laser beam axis has also been investigated.

\section{Separation of electrons and ions in laser-induced plasma}

Certain experimental evidences by Stuart et al (1996) and Tonshoff et al (2000) reveal that, the kinetic energy of the ions in the laser-induced plasma increases with charge. These observations have lead to development of a model to explain the large translational energy of ions. In these models, electrons, being the lighter particles with higher velocities, were assumed to escape from the plasma. This is shown in figures 1 and 2 . This causes formation of a negatively charged sheath around the plasma. The electrostatic interaction between the negative sheath and the positive plasma would accelerate the ions. The resulting mean ion energy $E_{i}$ is given 


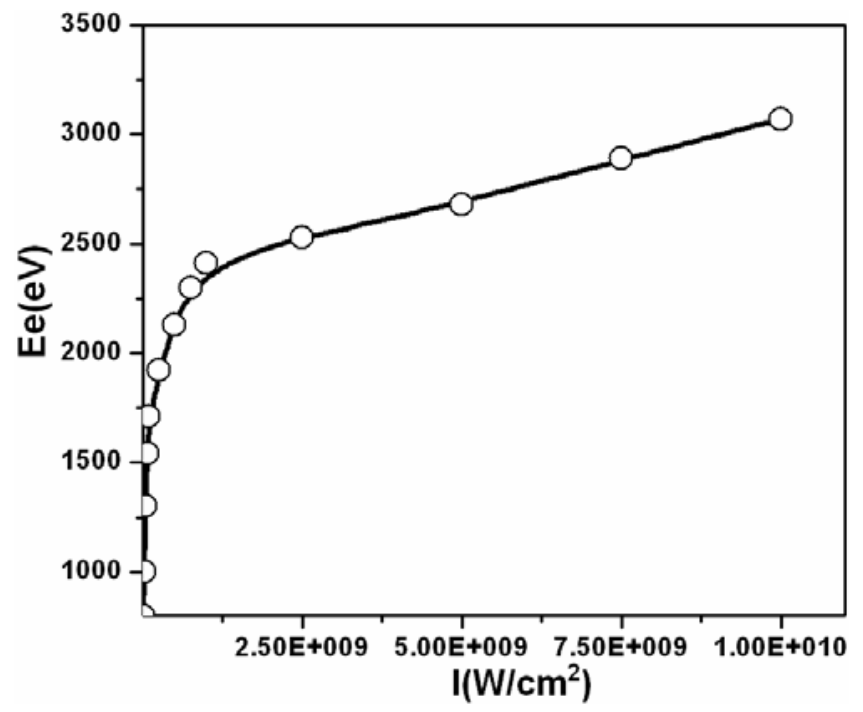

Figure 1. Laser intensity is the function of electron energy distribution in the laser-induced plume with given parameters; $t=5 \mathrm{~ns}$; $\lambda=1064 \mathrm{~nm} ; P=0.7 \mathrm{~Pa}$; $E=3 \mathrm{~J}$.

by the following equation:

$$
E_{i} \approx(Z+1) k_{B} T,
$$

where $Z$ is the charge multiplicity, $T$ is the initial temperature of the plasma and $k_{B}$ is the Boltzmann's constant.

A Nd: YAG pulsed laser operating at $1064 \mathrm{~nm}, 5-10 \mathrm{~ns}$ pulse width, pulse energy up to $900 \mathrm{~mJ}$ and $10 \mathrm{~Hz}$ repetition rate, was employed to irradiate metallic Ti target to produce intense flow of highly charged ions which constitutes the non-equilibrium plasmas. The high power density of the laser light, of the order of $10^{10} \mathrm{~W} / \mathrm{cm}^{2}$, produces high energy nonequilibrium plasma at the target surface. The high density and high temperature of the plasma induces formation of highly ionised Rydberg states with charge contents close to $10^{+}$in heavy elements. These ions are also associated with kinetic energy of the order of $1-50 \mathrm{eV}$. The ion

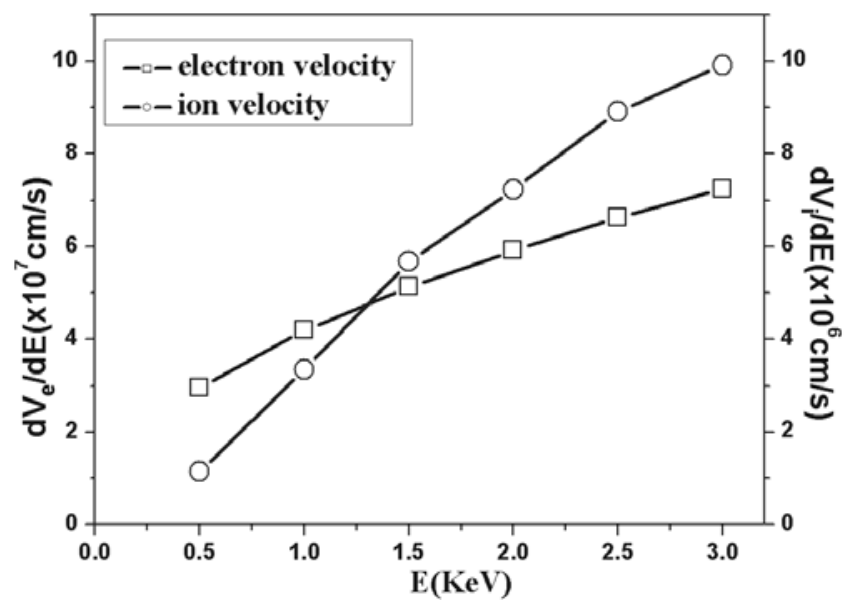

Figure 2. Energy of electron and ion versus electron and ion velocity $\lambda=1064 \mathrm{~nm} ; P=0.7 \mathrm{~Pa}$; $I=10^{8} \mathrm{Wcm}^{-2}$. 


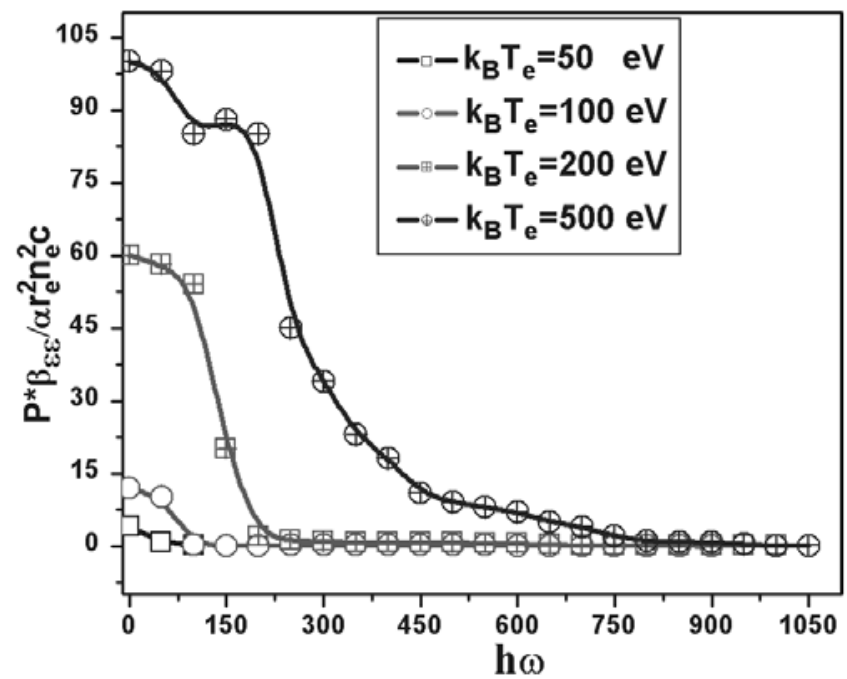

Figure 3. Electron-electron Bremstrahlung emission in plasma for four values of $k_{B} T_{e} ; t=5 \mathrm{~ns}$.

emission is accompanied by a neutral emission. The extent of fractional ionisation depends on the nature of the target and is generally of the order of 2 to 20\% (Stuart et al 1996). Ion and neutrals are ejected mainly along the axis normal to the target surface.

Electric and magnetic fields can be employed to inject the ions into an intense plasma source with high efficiency. By using $10 \mathrm{~Hz}$ repetition rate it has been calculated from the experimental data that a maximum injectable ion current up to $2 \mathrm{~mA}$ as reported by Vorobev (1993) and Zeldovich \& Raizer (1967).

The experimental evidence indicating the formation of accelerating dusty plasma from the material during the time of the laser pulse has led to formulation of a model which invokes the concept of absorption of laser radiation for material evaporation. The mechanism prevailing for the absorption is the inverse electron-electron Bremsstrahlung emission in plasma. This is presented in figure 3 for four different values of the electron temperature. The thermal energy of the material, however, can be an order of magnitude lower than the energy corresponding to the maximum velocity of the expanding plasma front. A large number of hydrodynamic treatments have been used to analyse the expansion of the heated materials as reported by Kumar (2001) and Vorobev (1993). In figure 3, power emitted per unit volume per unit energy interval $P * \beta_{e e}$ are in units of $\alpha r_{e}^{2} n_{e}^{2} c$ is plotted against photon energy $\eta w$. Here $\alpha=e^{2} / 2 \eta c \varepsilon_{0}$ is the fine structure constant and $r_{e}=4 \pi c^{2} m_{e} \varepsilon_{0}$ is classical electron radius. This figure also depicts electron-electron Bremstrahlung emission in plasma for four different values of $k_{B} T_{e}$. These results are for $5 \mathrm{~ns}$ laser pulse.

A treatment for expansion of one-dimensional plasma proximal to specimen surface $(x=0$, $t=0$ ) is dealt here. Physically, pressure gradient within the plasma drives its expansion. A density gradient is considered in the present model. The pressure gradient in the plasma is physically constructed as follows

$$
P=P s\left(1-\frac{x}{X}\right), \quad x \leq \stackrel{*}{X} .
$$

In this equation, $P$ is the driving pressure, $P_{s}$ is the radiation pressure at material surface and $\stackrel{*}{X}$ is the distance of the plasma edge from the specimen surface. The results of the 
various models turn out to be similar, independent of the nature of density gradient employed (Stapleton \& Mosnier 2002).

For sake of brevity, radiation losses are neglected. The rate $W$ at which the laser beam deposits energy in the plasma equals the rate at which the plasma does work in its expansion plus the rate at which the thermal energy of the plasma increases (Stoian et al 2000). For an one-dimensional plasma having cross sectional area $A$, expanding outwards from the specimen surface, the mathematical expression can be written as

$$
-A \int_{0}^{\stackrel{*}{X}} \frac{\partial P}{\partial x} \frac{d x}{d t} d x+\frac{1}{2} A \frac{d}{d t} \int_{0}^{\stackrel{*}{X}}\left(n_{i}+n_{e}\right) k_{B} T d x
$$

where,

$$
W=I \times A \int_{0}^{x} K_{v} \exp ^{-K_{v} x} d x
$$

The absorption coefficient $K v$ can be derived as

$$
\left\{\begin{array}{rl}
K_{v} & =\left(\frac{4}{3}\right)\left(\frac{2 \pi}{3 k_{B} T}\right)^{1 / 2}\left(n_{e} n_{i} Z^{2} e^{6} / \eta \mathrm{cm}_{e}^{3 / 2}\right)\left[1-\exp \left(-\frac{\eta \gamma}{k_{B} T}\right)\right] \\
& =3.69 \times 10^{8}\left(\frac{Z^{3} n_{i}^{2}}{T^{1 / 2}} \gamma^{3}\right)\left[1-\exp \left(-\frac{\eta \gamma}{k_{B} T}\right)\right]
\end{array},\right.
$$

where $n_{i}$ and $n_{e}$ are electron and ion densities in the laser derived plasma having average charge $Z$ and temperature $T, \gamma$ is the frequency of the laser beam, $c, e, m_{e}, \eta$ and $k_{B}$ are the velocity of light, electronic charge, electron mass, Planck's constant and Boltzmann's constant, respectively, $I$ represents laser irradiance.

The equation of state for plasma can be expressed as

$$
P=\left(n_{i}+n_{e}\right) k_{B} T .
$$

The spatial variations of electron and ion densities in laser-induced plasma are given as follows

$$
n_{i}=n_{i s}\left(1-\frac{x}{X}\right) ; \quad n_{e}=n_{e s}\left(1-\frac{x}{X}\right),
$$

where $n_{i s}$ and $n_{e s}$ denote the ion and electron densities at the surface of the specimen material, respectively. If we assume a similarity expansion (time invariant density profile), then the velocity of the plasma increases linearly with the distance from the surface of the material derives as follows

$$
\frac{d x}{d t}=\left(\frac{x}{\underset{X}{*}}\right) \frac{d x}{d t}
$$

Inserting equations (2), (6) and (7) in the left hand side of equation (3), following new equation can be obtained

$$
\left\{\begin{array}{c}
A \int_{0}^{\stackrel{*}{X}} \frac{P s x d x}{\stackrel{* 2}{X} d t} d x+\frac{1}{2} A \frac{d}{d t} \int_{0}^{\stackrel{*}{X}}\left(n_{i s}+n_{e s}\right)\left(1-\frac{x}{\stackrel{*}{X}}\right) k_{B} T d x \\
=A \frac{P s}{2} \frac{d X}{d t}+\frac{1}{2} A \frac{d}{d t}\left(n_{i s}+n_{e s}\right) \frac{*}{2} k_{B} T=W
\end{array}\right.
$$




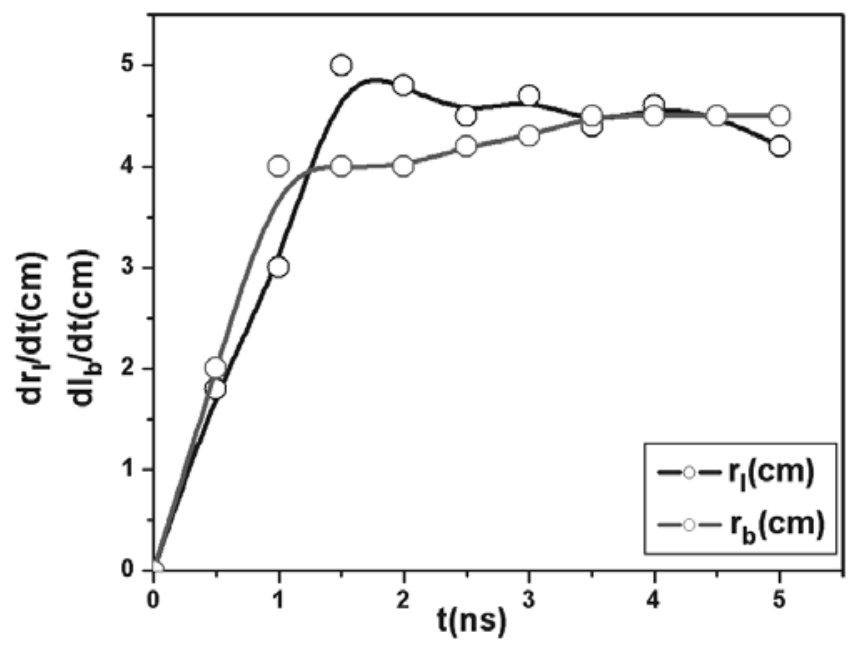

Figure 4. Laser pulse interaction duration is the function of growth rate of radial and axial plume dynamics without external magnetic field, when $\lambda=$ $1064 \mathrm{~nm} ; P=0.7 \mathrm{~Pa} ; I=$ $10^{8} \mathrm{Wcm}^{-2}$.

In a thermally conducting plasma, $T$ is independent of spatial position. Then the total number of ions $N_{i}$ is given by Samarsky \& Popov (1975)

$$
N_{i}=A \int_{0}^{\stackrel{*}{X}} n_{i s}\left(1-\frac{x}{\underset{*}{X}}\right) d x=A n_{i s} \frac{\stackrel{*}{2}}{2},
$$

with insertion of similar expression for the total number of electrons $N_{e}$, the equation (8) assumes the following form:

$$
\left(A \frac{P s}{2}\right) \frac{d}{d x}+\frac{1}{2} k_{B}\left(N_{i}+N_{e}\right) \frac{d T}{d t}=W .
$$

The rate at which work is done by the plasma during its expansion equals the rate of increase of plasma kinetic energy. It has been shown that the plasma propagating away from the material surface has an elliptical configuration owing to non-uniform distribution of pressure inside the plasma. These computational results are shown in figure 4 . This is the maximum in the axial direction due to maximum hydrodynamic pressure exhibited towards this direction. The energy balance yields the following expression:

$$
-A \int_{0}^{*} \frac{\partial P}{\partial x} \frac{\partial x}{d t} d x=\frac{1}{2} \frac{d}{d t} \int_{0}^{*}\left(n_{i} m_{i}+n_{e} m_{e}\right) A\left(\frac{d x}{d t}\right)^{2} d x
$$

Substituting equations (5)-(8) and (9), the integration yields in following form

$$
\left(\frac{A P s}{2}\right) \frac{d \stackrel{*}{X}}{d t}=\frac{1}{12}\left(\frac{d}{d t}\right)\left(\frac{d x}{d t}\right)^{2}\left(N_{i} m_{i}+N_{e} m_{e}\right),
$$

and substituting equations (2), (5) and (6) into (12) gives

$$
\left\{\begin{aligned}
\frac{\left(N_{i}+N_{e}\right)}{A \stackrel{*}{X}} \frac{d \stackrel{*}{X}}{d t} & =\frac{1}{12} \frac{\left(N_{i} m_{i}+N_{e} m_{e}\right)}{A} \frac{d}{d t}\left(\frac{d \stackrel{*}{X}}{d t}\right)^{2}, \\
& =\frac{1}{6} \frac{\left(N_{i} m_{i}+N_{e} m_{e}\right)}{A} \frac{d \stackrel{*}{X}}{d t} \frac{d^{2} X}{d t^{2}}
\end{aligned}\right.
$$


or,

$$
T=\left[\left(N_{i} m_{i}+N_{e} m_{e}\right) \frac{\stackrel{*}{X}}{6 k}\left(N_{i}+N_{e}\right)\right] \frac{d^{2} \stackrel{*}{X}}{d t^{2}} .
$$

The results from these calculations obtained for velocity of the plasma edge are in conformity with the published experimental data by Anisimov \& Lukyanchuk (2002). It is shown in figure 4 that during the laser pulse duration, the velocity of the luminous edge increases along with the increase in value of the average energy until the plasma becomes transparent.

The plasma temperature first increases due to intense rate of absorption of the laser radiation by the material. At some instant of time (the time at which average particle energy stops increasing) the radius of the plasma starts increasing which causes an exponential drop in rate of energy absorption. Thus the plasma temperature drastically decreases. The total energy of plasma is used as kinetic energy for the radial expansion. It is important to note that the temperature of the material is not well derived from a simple measurement of the time of flight (Capitelli et al 2004); (Belotserkovsky \& Davidov 1982). The high velocities obtained are associated with the directed motion of the center of mass of the ions rather than thermal energy. It clearly appears that the atoms are removed early during the laser pulse schedule. However, the material is heated and reaches a temperature of the order of $300-600 \mathrm{eV}$. Following this, it undergoes an expansion into the vacuum and becomes transparent at some stage where the particle density is sufficiently low.

For the three-dimensional expansion, with linear radial density gradient and under the assumption that small number of particles are there in the target, the equation analogous to (13) can be expressed as:

$$
\frac{d^{3}}{d t^{3}\left(R^{2}\right)}=\frac{10 W}{\left(N_{i} m_{i}+N_{e} m_{e}\right)} .
$$

The temperature of the plasma is also derived as

$$
T=\frac{2}{15}\left[\left(N_{i} m_{i}+N_{e} m_{e}\right) \frac{R}{\left(N_{e}+N_{i}\right)} k_{B} T\right] \frac{d^{2} R}{d t^{2}} .
$$

where $R$ is the radius of the expanding plasma drop.

\section{Physics of laser-induced plasma expansion}

Since the laser intensity exceeds the ablation threshold of the target, the laser beam causes evaporation and ionization of material creating a plasma plume above the material surface. Initially the atoms, molecules and ions undergo collisions in the high-density region near the target forming the so called Knudsen layer. This leads to a highly directional expansion of the plasma plume perpendicular to the target. The expansion dynamics of the laser-produced plasma plume have been described elsewhere in a semi-quantitative model propounded by Anisimov et al (1974) and Drogoff et al (2004). In the initial stage, the interaction of the laser beam with the bulk target results in the evaporation of the surface layer. Further interaction of the laser beam with the evaporating material leads to formation of an isothermally expanding plasma. This expansion persists until the termination of the laser pulse. The vapor particles 


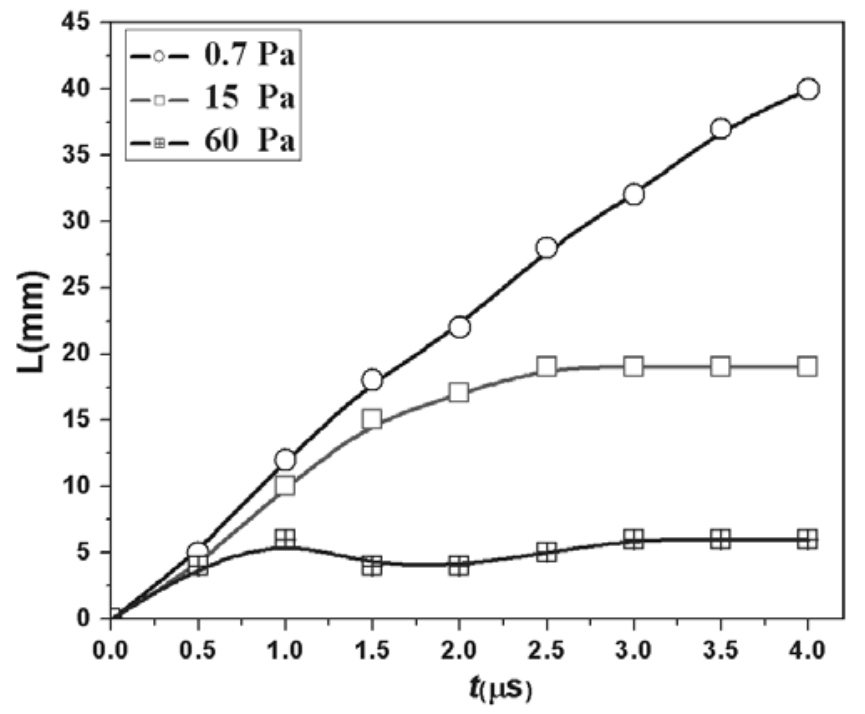

Figure 5. Plume length with different pressures, in $(\mathrm{Pa})$, when $t=5 \mathrm{~ns} ; \lambda=1064 \mathrm{~nm} ; I=$ $10^{8} \mathrm{Wcm}^{-2}$.

are pushed forward in the lateral direction exclusively by the high pressure emanating from the target surface when the ejected plume is considered transparent to the incident laser beam. Strong laser-plasma interaction creates an additional high-pressure kinetic energy region fuelling further expansion of the plume. After the termination of the laser pulse, no further particles are ejected from the target surface. An adiabatic expansion of the plasma occurs where the temperatures can be related to the calculated dimensions of the plasma shown in figure 4. It is found from the present calculations that the forward directed nature of the laser evaporation process results from anisotropic expansion velocities of different species governed by the initial dimensions of the expanding plasma. The thermal energy is rapidly converted into kinetic energy with the plasma attaining extremely high expansion velocity. During the initial stages of plasma expansion, when the particle density is high $\left(\sim 10^{19} \mathrm{~cm}^{-3}\right)$, the mean free path of the particles is short $(\sim \mu \mathrm{m})$ and the plasma behaves as a continuum fluid. As the plasma expands, the temperature drops very rapidly (within $100 \mathrm{~ns}$ time). However, the rate of temperature drop is smaller at later times ( $>100 \mathrm{~ns}$ ) because energy is regained due to recombination of ions.

The plasma, expanding freely in vacuum, is shown in figure 5. As the background pressure increases, the plume behaviour is characterized by strong interpenetration of the laser plasma by ambient low density gas. The expansion dynamics of the plume in this pressure regime is determined by the properties of the plasma as well as the prevailing background gas. In this regime, collisional effects start to play a role as discussed by Mao et al (2000); Russo (1995) and Singh et al (1990). At still higher background pressures, the expansion dynamics of the plasma are fully governed by the pressure of the ambient gas as shown in figure 6. In general, an increase in background pressure results in the following effects as reported by Gornushkin et al (2001) and Mazhukin et al (2002): (i) an increase in fluorescence from all the species due to enhanced collisions on the expansion front and subsequent inter-plume collisions, (ii) shock front formation, (iii) slowing down of the plume compared to its propagation in vacuum. The last effect is caused by spatial confinement of the plasma. The presence of ambient gas during expansion may also lead to reactive scattering, thermalization of the plume and enhanced condensation leading to cluster generation with possibilities for nanoparticle formation. 


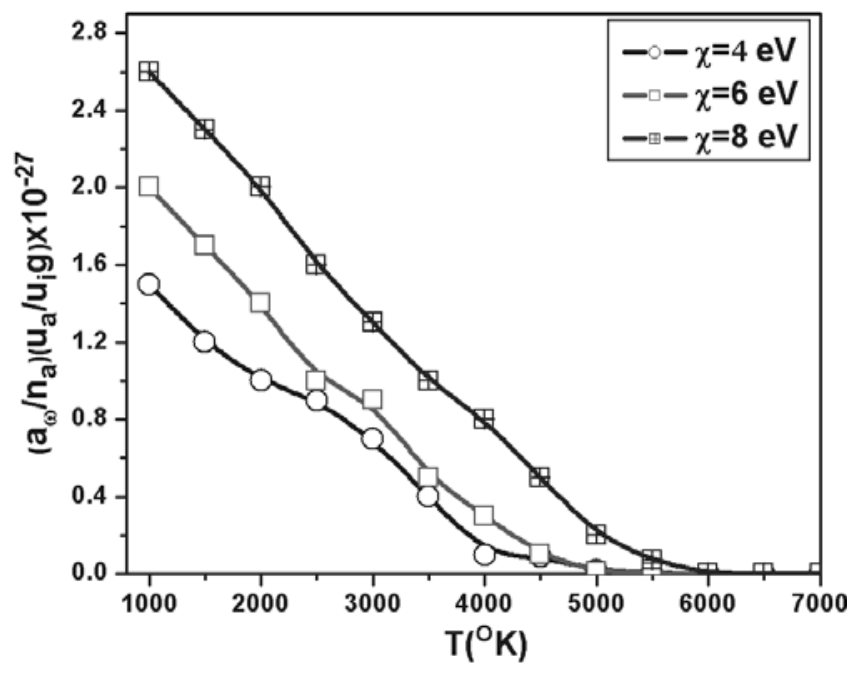

Figure 6. Temperature dependence function determining the rate of heating of slightlyionized plasma by electron-ion Bremsstrahlung.

\section{Plume length}

Using an adiabatic expansion model, numerical calculation was carried out to estimate the length of the plume at different ambient gas pressures in accordance with the procedure reported by Mazhukin et al (2002). The ranges of $\mathrm{Ar}^{+}$ions were also determined. According to this model, the ablated species push the background gas species until the plasma and gas pressures are equilibrated. Then the length of the plasma is given by

$$
L=A_{L}[(\gamma-1) E]^{1 / 3 \gamma} P_{0}^{-1 / 3 \gamma} V^{\gamma-1 / 3 \gamma}
$$

where $A_{L}$ is the geometrical factor related to the shape of the laser spot at the target surface, $\gamma$ is the ratio of specific heat, $E$ is the laser energy, $P_{0}$ is the ambient gas pressure, $V$ is the initial volume of the plasma expressible as $V=v_{0} \tau w$ (where $v_{0}$ is the initial species velocity, $\tau$ is the laser pulse width and $w$ is the spot size at the target surface). The factor $A_{L}$ depends on the expansion geometry. For a conical plume element, its dependence on expansion angle $\theta$ is given by the equation

$$
A_{L}=\left[1+\frac{1}{\tan \theta}\right]\left[\frac{3 \tan \theta}{\pi+2 \pi \tan \theta}\right] .
$$

Considering experimental parameters with $\theta=45^{\circ}$, the estimated values of plume length at different pressures along with experimental maximum range of the plume, are given in the figure 5. This is fairly in good agreement with the experimentally observed plume lengths obtained from CCD imaging carried out by Russo (1995). Both the experiment and the model predictions reveal that the ambient gas slows down the expansion of the ablated plume.

The estimated stopping distance is in good agreement with experimentally observed value of plume length at pressure $\sim 0.7 \mathrm{~Pa}$. However, these calculations did not agree to the experimental finding of plume lengths at higher pressures. These lengths were found to be much higher when compared with the calculated $\mathrm{Ar}^{+}$ion range. Thus, it is established that the present numerical calculations are effective only at lower pressures. 


\subsection{Thermal ionization of the plasma}

The temperature in the hottest region of the plasma generated by the laser pulse, rises as long as the local rate of the absorption of energy from the beam is sufficient enough to overcome cooling by expansion and conduction. Similar inferences have been discussed by Rethfeld et al (2004) and Furusawa et al (2004). Eventually, the plasma may attain sufficiently high temperature to cause ionization of significant number of atoms through collisions. Assuming persistence of thermodynamic equilibrium for a sufficiently dense vapour, Saha's equation can be invoked to relate electron $\left(n_{e}\right)$, ion $\left(n_{i}\right)$ and neutral atom densities $\left(n_{a}\right)$ at a given temperature $T$. The equation is laid down as follows

$$
\frac{n_{e} n_{i}}{n_{a}} \cong \frac{2 u_{i}}{u_{a}}\left(\frac{2 \pi m_{e} k_{B} T}{h^{2}}\right) \exp \left(\frac{-\chi}{k_{B} T}\right),
$$

where $u_{i}, u_{a}$ are the partition functions for the singly-ionized and neutral atoms, respectively. $\chi$ is the ionization energy of the neutral atoms, $k_{B}$ is the Boltzmann's constant. The net absorption coefficient for inverse Bramsstrahlung of frequency $\omega$ can be written in terms of the equation reported by Anisimov et al (1974) and Anisimov \& Lukyanchuk (2002)

$$
a_{\omega} \cong \frac{n_{e}^{2} e^{6} \bar{g}}{6 \varepsilon_{0}^{3} c \eta \omega^{3} m_{e}^{2}}\left(\frac{m_{e}}{6 \pi k_{B} T}\right)^{1 / 2}\left[1-\exp \left(-\frac{\eta \omega}{k_{B} T}\right)\right],
$$

where $\bar{g}$ is the average gaunt function. The dispersion term is assumed to be close to unity. Non-linear effects are not considered due to the fact that all positive ions are singly charged. The value of $n_{e}^{2}$ found from Saha's equation (18) is inserted into equation (19) to yield

$$
a_{\omega} \cong \frac{n_{a} e^{6} g}{12 \sqrt{3} \pi^{2} \varepsilon_{0}^{3} c \eta^{4} \omega^{3}} \frac{u_{i}}{u_{a}} k_{B} T \exp \left(-\frac{\chi}{k_{B} T}\right)\left[1-\exp \left(-\frac{\eta \omega}{k_{B} T}\right)\right] .
$$

For a laser beam irradiance $10^{8} \mathrm{~W} / \mathrm{cm}^{-2}$, the power absorbed per unit area of the beam across a depth $l$ of the laser plasma is expressed as $I\left[1-\exp \left(-a_{\omega} l\right)\right]$. Subjected to the condition that $l \ll 1 / a_{\omega}$, the absorbed power can be approximated. The mean absorption rate per unit volume in this region is therefore taken as $I a_{\omega} l$. Given that the degree of ionization is not too large, the total number of particles per unit volume will not exceed $n_{a}$. While in thermal equilibrium, the mean power absorbed per particle, $P$ becomes $I a_{\omega} / n_{a}$. As seen from the equation (20), $P$ is independent of density and increases with temperature. This continues as long as the degree of ionization remains low. In figure 6 , the quantity $\left(\frac{a_{\omega}}{n_{a}}\right)\left(\frac{u_{a}}{u_{i} \bar{g}}\right)$ in $\mathrm{cm}^{-2}$, is plotted as a function of temperature for three values of the ionization energy $\chi$. The rate of temperature rise due to this process is given by the $I a_{\omega} / n_{a} K_{B} \mathrm{Ks}^{-1}$, where $k_{B}$ is Boltzmann's constant expressible in term of Joules $\mathrm{K}^{-1}$. As an example, consider a vapour with an ionization energy $8 \mathrm{eV}$ at a temperature of $6000 \mathrm{~K}$. Taking $u_{a} / u_{i} \bar{g} \approx 1, a_{\omega} / n_{a}$ is found to be about $2 \cdot 10^{-27} \mathrm{~cm}^{-2}$. The rate of temperature rise in $\mathrm{Ks}^{-1}$ numerically equals the irradiance in $\mathrm{W} / \mathrm{cm}^{-2}$. As the temperature rises, the rate of heating increases with intensity. The vapor absorbed per particle begins to fall with the increasing temperature and becomes density dependent.

\section{Black body radiation of laser-induced plasma}

If the plasma is in local thermodynamic equilibrium (LTE), the radiation within it has a well defined frequency distribution. The energy density of LTE plasma clearly depends on 
temperature $T$. Under these circumstances, Planck function for a single polarization event can be expressed in accordance with the equation propounded by Anisimov et al (1974):

$$
w_{\omega B_{0}}=\frac{\eta \omega^{3}}{2 \pi^{2} c^{3}}\left[\exp \left(\frac{\eta \omega}{k_{B} T}\right)-1\right]^{-1} .
$$

For a dispersive medium, the Planck function $\gamma_{\omega B}(T)$ at the black body temperature $T$ is expressed as follows

$$
\gamma_{\omega B}(T) \cong \frac{\mu^{2} \eta \omega^{3}}{8 \pi^{3} c^{2}}\left[\exp \left(\frac{\eta \omega}{k_{B} T}\right)-1\right]^{-1}
$$

for long wavelength, such that $\eta \omega \ll k_{B} T$, the Raleigh-Jeans law is given as:

$$
\gamma_{\omega B}(T) \cong \frac{\mu^{2} \omega^{2} k_{B} T}{8 \pi^{3} c^{2}}
$$

The spectral emittance per unit angular frequency for a single polarization event is given by:

$$
M_{\omega B_{0}} \cong \frac{c}{4} w_{\omega B_{0}} \cong \frac{\eta \omega^{3}}{8 \pi^{2} c^{2}}\left[\exp \left(\frac{\eta \omega}{k_{B} T}\right)-1\right]^{-1},
$$

or, in the terms of the wavelengths $\lambda$, per unit wavelength interval, the expression becomes

$$
M_{\lambda B_{0}} \cong \frac{2 \pi \eta c^{2}}{\lambda^{5}}\left[\exp \left(\frac{\eta c}{\lambda k_{B} T}\right)-1\right]^{-1}
$$

Numerically, the electron wavelength in plasma is expressed in centimeters and temperature in Celsius. These are presented in figure 7. This reveals that the electron wavelength increases logarithmically with the decreasing electron temperature in the plasma. This occurs due to electron-electron collision effect taking place inside plasma.

$$
M_{\lambda B_{0}} \cong \frac{3.74 \times 10^{8}}{\lambda^{5}}\left[\exp \left(\frac{1.43 \times 10^{4}}{\lambda k_{B} T}\right)-1\right]^{-1} \mathrm{Wm}^{-2} \mu \mathrm{m}^{-1}
$$

\subsection{Laser-induced plasma model}

The emission of laser-induced plasma is determined by the combined effect of many processes. Both collisional/radiative, excitation/de-excitation and ionization/recombination phenomena can occur. These events can involve multiple energy levels and several stages of ionization. We shall consider briefly three plasma models. It is assumed that the free electrons have a Maxwallian distribution at temperature $T_{e}$, determined by external conditions laid down by Anisimov et al (1974) and Rethfeld et al (2004). Thus the number of electrons, with velocity spread between $u$ and $u+d u$ is given by the following expression

$$
d n_{e}(u) \cong 4 \pi n_{e}\left(\frac{m_{e}}{2 \pi k_{B} T}\right)^{1 / 2} \exp \left(\frac{-m_{e} u^{2}}{2 k_{B} T_{e}}\right) u^{2} d u .
$$




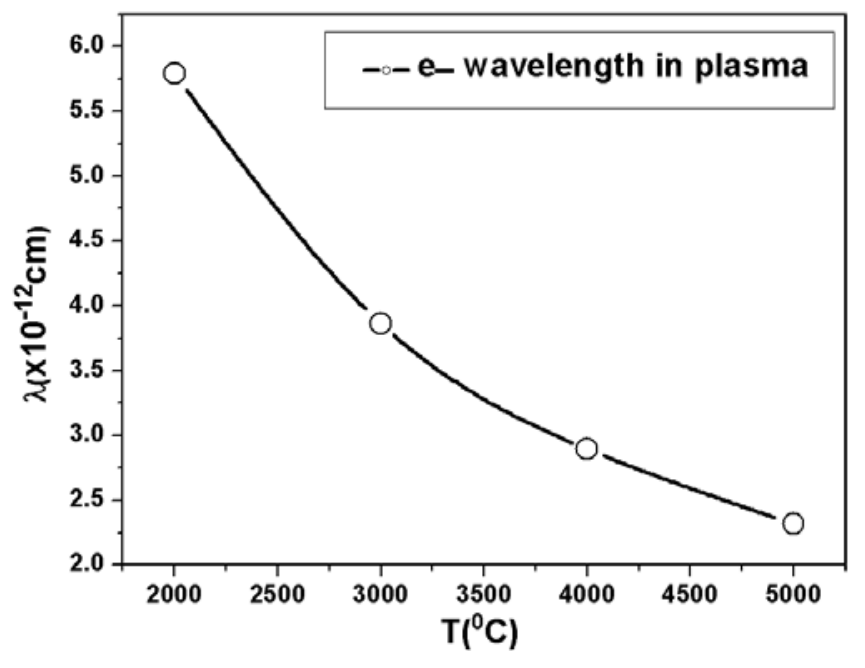

Figure 7. Temperature of electron is the function of electron's wavelength in laser-induced material plasma, when $t=5 \mathrm{~ns}$; $E=4 \mathrm{~J} ; \lambda=1064 \mathrm{~nm}$; $I=10^{8} \mathrm{Wcm}^{-2}$.

\subsection{Local thermodynamic equilibrium (LTE)}

Collisions become more frequent as the density of plasma is increased. At sufficiently high densities, collision processes determine excited state populations. The LTE model of the laserinduced plasma assumes that population of atoms and ions in the various bound states are controlled entirely by electron collision, thus enabling their determination from the principle of equipartition. For this condition, plasma structure should not change rapidly. Thus, for the population $n_{1}$ and $n_{2}$ of two bound states 1 and 2 of the atom or ion, with energies $\omega_{1}$ and $\omega_{2}$, the Boltzman's equation stated as:

$$
\frac{n_{1}}{n_{2}} \cong \frac{g_{1}}{g_{2}} \exp \left(\frac{\omega_{2}-\omega_{1}}{k_{B} T_{e}}\right)
$$

The densities $n_{Z}, n_{Z+1}$ of the ground state atoms or ions of the charge $Z+1(Z=0,1 \ldots)$ are related by Saha's equation (5)

$$
\frac{n_{e} n_{Z+1}}{n_{Z}} \cong \frac{2\left(g_{0}\right)_{Z+1}}{\left(g_{0}\right)_{Z}}\left(\frac{2 \pi m_{e} k_{B} T_{e}}{\eta^{2}}\right) \exp \left(\frac{-\chi_{Z}}{k_{B} T_{e}}\right) .
$$

Here $\left(g_{0}\right)_{Z}$ is the statistical weight of the ground state of an atom or ion with charge $Z$ and $T_{e}$ is the electron temperature. The set of equation for all pairs of adjacent stages of ionization together with the total density of nuclei determine the equilibrium population of the ionized species completely. For the LTE model to apply, the plasma electron density must be sufficiently high for effective collisional de-excitation to occur. It should be at least 10 times more probable than the radiative decay for all transitions. In optically thin plasma, it may be expressed as:

$$
n_{e} \geq 1.6 \times 10^{12} T_{e}^{1 / 2}(\Delta W)_{\max }^{3} \mathrm{~cm}^{-3},
$$

where $(\Delta W)_{\max }$ is the largest energy interval between adjacent energy levels of atoms and ions in the plasma in eV. Units $T_{e}$ is expressed in $K$. In optically thick plasma, the LTE model is valid at lower densities with $(\Delta W)_{\max } \leq 0 \cdot 8 \chi$, for all atoms and ions, the lower limit to 
$n_{e}$ for LTE conditions, is given by the identity

$$
n_{e} \geq 8 \times 10^{11} T_{e}^{1 / 2}(\Delta W)_{\max }^{3} \quad \mathrm{~cm}^{-3},
$$

where $x_{\max }$ refers to the the highest ionization energy of any atom or ion present in the plasma.

\subsection{Coronal equilibrium}

Collisional ionization is balanced by the radiative decay from the upper level to the lower levels. Rates of both these processes are proportional to the electron densities. Considering ionization and recombination effects, the ratio of number density of ions carrying charge $Z$ and $Z+1$, is given by the equation proposed by the Mazhukin et al (2002):

$$
\frac{n_{Z}}{n_{Z+1}} \approx 8 \times 10^{-9} \chi_{Z}^{1 / 4}\left(k_{B} T_{e}\right)^{-3 / 4} \exp \left(\frac{\chi_{Z}}{k_{B} T_{e}}\right) .
$$

The validity of above coronal model is subjected to two conditions. On one hand, the electron density must be sufficiently low and on the other hand, collisional deactivation must proceed at slower pace than the radiative decay. As the principal quantum number $n$ increases, the probability of spontaneous radiation is declined but the probability of a collosional transition is enhanced. Hence the coronal model breaks down at higher quantum numbers.

$$
n_{e} 6 \times 10^{8}(Z+1)^{6} T_{e}^{1 / 2} \exp \left[\frac{1 \cdot 62 \times 10^{3}(Z+1)^{2}}{T_{e}}\right] \mathrm{cm}^{-3},
$$

where $T_{e}$ refers to electron temperature in Kelvin.

\subsection{Spherical plasma with uniform temperature}

Although calculations on laser plasma dynamics were first presented by Anisimov et al (1974), the physical structure and associated phenomena still remains complicated. The target is initially supposed to be small, spherical, cool from which a fully ionized plasma of radius $r_{0}$ and mass $M$ at a critical electron density of $n_{e c}$ gets generated. The temperature $T$ and particle densities $n_{e}, n_{i}$ within the plasma volume are assumed to be uniform (Stoian et al 2000). The laser energy is supposed to be delivered to the plasma at a constant rate $P_{0}$. The radiation losses are neglected. The work done by plasma pressure $P_{i n j}$ in expanding the plasma is taken to be equal to the increase in kinetic energy of radial motion of the plasma. Corresponding radius of plasma boundary is $r_{b}$. For an isotropic expansion of the plasma, the governing equation can be written as

$$
4 \pi r_{b}^{2} \frac{d r_{b}}{d t} P \cong \frac{1}{2} \bar{M}-\frac{d}{d t}\left[\frac{d r_{b}}{d t}\right]^{2}
$$

where $\bar{M}$ refers to average value of plasma mass. Above expression allows the existence of radial velocity gradient during plasma expansion. Assuming uniform plasma density prevailing throughout, the average plasma mass can be written as $\bar{M}=3 M / 5$. Then the expression for plasma pressure is written as

$$
P \cong\left(n_{e}+n_{i}\right) k_{B} T
$$




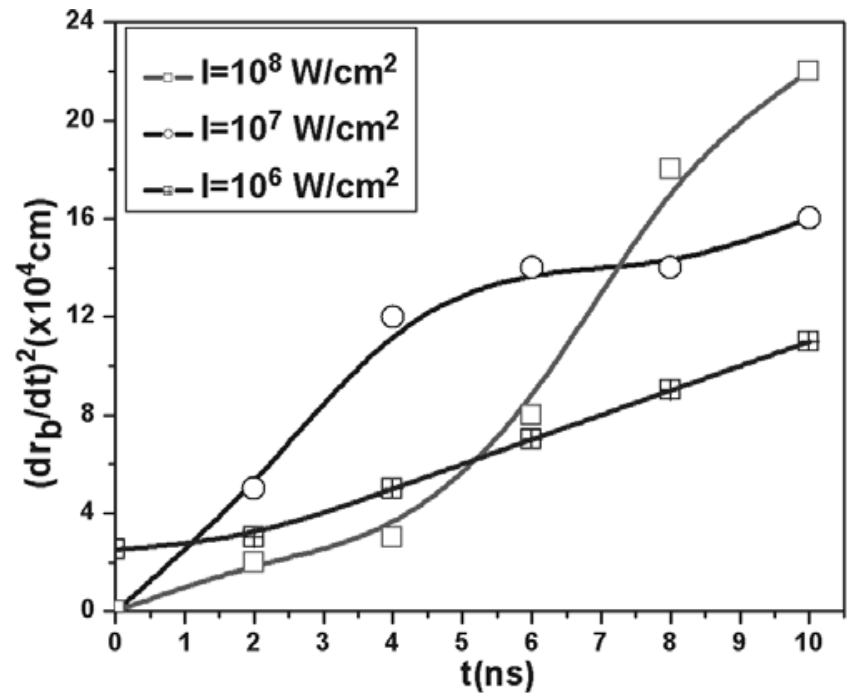

Figure 8. Laser pulse is function of radial plume dynamics for three values of laser intensity; with $\lambda=$ $1064 \mathrm{~nm} ; P=0.7 \mathrm{~Pa}$.

The mass of electrons is negligible as compared to the ion mass. The ion density can be expressed as

$$
n_{i} \cong \frac{3 M}{4 \pi r_{b}^{3} m_{i}}
$$

If $Z$ is the average charge associated with a positive ion, the charge balance leads to the condition $n_{e} \approx Z n_{i}$. In this case, the total number of particles in the plasma can be described by

$$
N \cong \frac{M(1+3)}{m_{i}}
$$

Some amount of the laser energy meets the energy cost of radial expansion. The rest goes to raising the temperature of the plasma. In figures 8 it is shown that increase in laser intensity enhances the rate of plasma expansion. This is due to introduction of higher magnitude of flux into the non-stationary plasma. It also causes time dependent volumetric expansion of the plasma. The axial expansion is three times higher compared to that in radial direction. The expression for plasma pressure is given by

$$
P_{0} \cong 4 \pi r_{b}^{2} \frac{d r_{b}}{d t} p+\frac{3}{2} N k_{B} \frac{d T}{d t},
$$

where $r_{b}$ refers to the radius of the plasma boundary. Setting $T=0$ and $\frac{d r_{b}}{d t}=0$ at $t=0$, the expression for radius of the plasma boundary becomes

$$
r_{b} \cong\left(r_{0}^{2}+\frac{10}{9} \frac{P_{0} t^{3}}{M}\right)^{1 / 2},
$$

where $r_{0}$ is the initial radius of the fully ionized plasma. The energy expression is obtained as

$$
k_{B} T \cong \frac{P_{0} t}{3 N}\left(\frac{2 r_{0}^{2}+\frac{5}{9}\left(\frac{P_{0} t^{3}}{M}\right)}{r_{0}^{2}+\frac{10}{9}\left(\frac{P_{0} t}{M}\right)}\right) \text {. }
$$




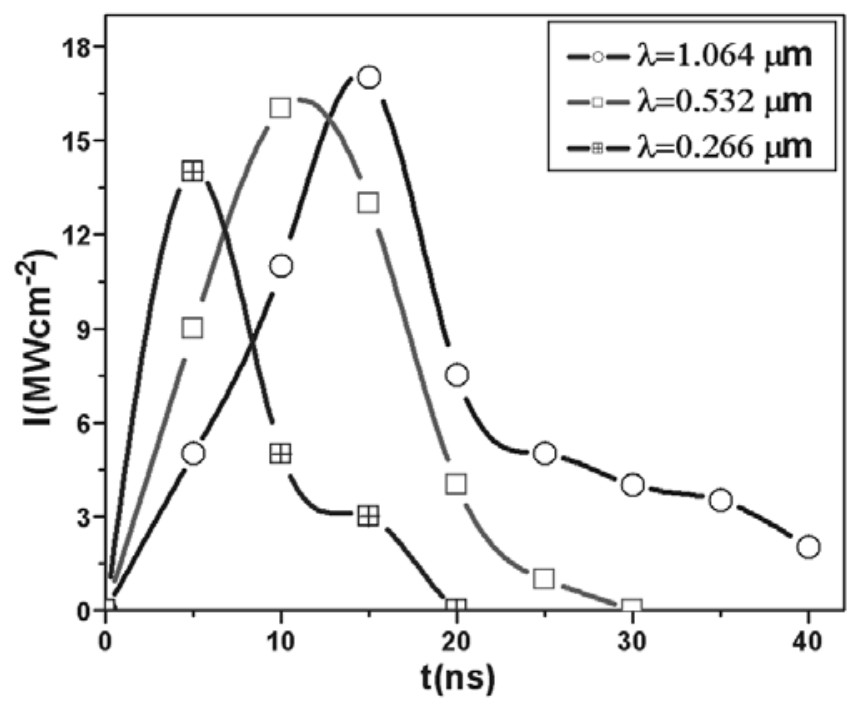

Figure 9. Laser intensity as a function of pulse width for three different values of laser wavelength.

For small values of $t,\left(t \ll\left(r_{0}^{2} M / P_{0}\right)^{3 / 2}\right)$, the above equation can be stated as

$$
\left\{\begin{array}{l}
r_{b} \approx r_{0}, \\
k_{B} T \approx \frac{2}{3} \frac{P_{0} t}{N} \quad(t \rightarrow 0) .
\end{array}\right.
$$

For large values of $t,\left(t \gg\left(r_{0}^{2} M / P_{0}\right)^{3 / 2}\right)$, the equation assumes the form

$$
\left\{\begin{array}{l}
r_{b} \approx\left(\frac{10}{9} \frac{P_{0} t^{3}}{M}\right)^{1 / 2} \quad(t \rightarrow \infty) . \\
k_{B} T \approx \frac{P_{0} t}{6 N}
\end{array}\right.
$$

Equation (41) indicates that for long duration laser pulses the thermal energy acquired by the plasma equals $3 N k_{B} T / 2$. This is only $1 / 4$ th of the total peak energy, $P_{o t}$, delivered by the different wavelengths of laser pulses shown in figure 9 . When the laser pulse ends or when plasma becomes transparent, no more energy is delivered to the plasma. It also describes average maximum temperature of the plasma after the end of the laser pulse for various values of laser wavelength. It is concluded that the time dependent growth of overall plasma temperature varies as square of the laser flux.

If it is assumed that if the heating is suspended suddenly at time $t_{h}$ when $T=T_{h}$ and $r_{b}=r_{h}$, the subsequent expansion and radius of the halted boundary is governed by the following expression

$$
\left\{\begin{array}{l}
T \cong T_{h}\left(\frac{r_{h}}{r_{b}}\right)^{2} \\
\left(\frac{d r_{b}}{d t}\right)^{2} \cong\left(\frac{d r_{b}}{d t}\right)_{t h}+\frac{5 N}{M} k_{B} T_{h}\left(1-\frac{r_{h}^{2}}{r_{b}^{2}}\right)
\end{array} \quad\left(t \geq t_{h}\right)\right.
$$

\subsection{Plasma in a magnetic field}

Highly conducting laser-induced plasma inside a magnetic field can only expand freely along the magnetic lines of force (Mao et al 2000); (Mazhukin et al 2002) and (Itina et al 2003). 
In the absence of diffusion, it must overcome the magnetic pressure for expansion in orthogonal direction. The energy $W$ required to produce a cavity of volume $V$ in a magnetic field $B$ is given by

$$
W \cong \frac{B^{2}}{2 \mu_{0}} V,
$$

$B$ is expressed in $K G, V$ in $\mathrm{cm}^{-3}$ and $W$ in Joules. $W$ can be written as $W \approx 4 \times 10^{-3} B^{2} V$. The structure of the plasma with uniform temperature and density that expands into the magnetic field assumes spherically symmetric geometry upon termination of the of the laser pulses. With repetitive laser pulses, the plasma periodically goes off the magnetic field. Maximum radius of boundary of this pulsating plasma is given by

$$
\left(r_{b}\right)_{\max } \approx\left(\frac{\mu_{0} N k_{B} T_{h}}{8 \pi B^{2}}\right)^{1 / 3} .
$$

The period of plume oscillation $\tau_{b} \approx \frac{\left(r_{b}\right)_{\max }}{V_{b \infty}} . N$ is the total number of particles, $T_{h}$ temperature at the end of the laser pulse and $V_{b \infty}$ is the asymptotic expansion velocity of the plasma. If the resistivity is finite and not too large, the plasma diffuses outwards across the magnetic field and the bounce radii increases with time. Sufficiently resistive plasma simply streams outwards without bouncing.

\section{Conclusion}

The average kinetic energy of the laser-induced plasma increases with charge. Such observations have led to formulation of a physical model for explaining large ion translational energies. In the present physical model, the negatively charged sheath around the plasma promotes electrostatic interaction with positively charged plasma. This accelerates the ions resulting in vastly enhanced ion energies. The experimental evidence shows acceleration of the laserinduced plasma into the material surface for short pulse duration. This causes absorption of laser radiation in the material leading to evaporation. The mechanism of energy absorption by the material is attributable to inverse Bremsstrahlung process. The energy associated with propagating plasma front in the direction of material surface is orders of magnitude higher than the energy absorbed by the material. In accordance with the assumptions of the present physical model neglecting the radiation losses, it can be undoubtedly stated that the rate of deposition of laser energy in the plasma equals the rate of which plasma does work for its expansion plus the rate of thermal energy increases. It is established from the present studies that plasma expands freely in vacuum i.e. at low background pressure alters the plume behaviour which is characterized by strong interpretation of the laser plasma and the ambient low density gas.

Theoretically, it is established that the temperature in the hottest region in the plasma generated by the laser pulse will rise as long as the local rate of the absorption of energy from the beam is sufficient to overcome the cooling by expansion and conduction. Eventually, the plasma may reach sufficiently high temperature needed for a significant number of atoms to be ionized by collisions.

The constitutive composition of laser-induced plasma is determined by the combined effects of many processes. Both collisional and radiative, excitation, de-excitation, ionization and recombination, involving many energy levels and several stages of ionization occurs. 


\section{Nomenclature}

\begin{tabular}{|c|c|}
\hline$E_{i}$ & Mean ion energy \\
\hline$Z$ & Electronic charge multiplicity \\
\hline$T$ & Initial temperature of plasma \\
\hline$k_{B}$ & Boltzmann's constant \\
\hline$P$ & Deriving pressure \\
\hline$P_{s}$ & Pressure of the material surface of the laser radiation \\
\hline$\stackrel{*}{X}$ & Distance of the edge of the plasma from the surface of the material \\
\hline$W$ & Laser beam power deposited in the plasma \\
\hline$A$ & Cross sectional area of plasma expanding outward from material surface \\
\hline$n_{i}, n_{e}$ & Electron and ion densities in the laser derived plasma \\
\hline$\gamma$ & Frequency of the laser beam \\
\hline$c, e, m_{e}, \eta, k_{B}$ & $\begin{array}{l}\text { Are the velocity of light, the electronic charge, electron mass, Planck's } \\
\text { constant and Boltzmann's constant, respectively }\end{array}$ \\
\hline$I$ & Laser irradiance \\
\hline$n_{i s}$ and $n_{e s}$ & Ion and electron densities at the surface of the material \\
\hline$N_{e}, N_{i}$ & Total number of electrons and ions \\
\hline$n_{e}, m_{e}$ & Density and mass of the electron \\
\hline$n_{i}, m_{i}$ & Density and mass of the ions \\
\hline$R$ & Expanding radius of the plasma drop \\
\hline$A_{L}$ & Geometrical factor related to the shape of the laser spot at the target surface \\
\hline$\gamma$ & Ratio of specific heat \\
\hline$E$ & Laser energy \\
\hline$P_{0}$ & Ambient gas pressure \\
\hline$V$ & Initial volume of the plasma \\
\hline$v_{0}$ & Initial velocity associated with ionized species \\
\hline$\tau$ & Pulse width of laser beam \\
\hline$w$ & Spot size at the target surface \\
\hline$u_{i}, u_{a}$ & Partition functions for the singly-ionized and neutral atoms, respectively \\
\hline$\chi$ & Ionization energy of the neutral atoms \\
\hline$\omega$ & Frequency of absorption coefficient for inverse Bremsstrahlung \\
\hline $\bar{g}$ & Average gaunt function \\
\hline$l$ & Beam depth of laser plasma \\
\hline$P$ & Mean laser power absorbed per particle \\
\hline$\gamma_{\omega B}(T)$ & Spectral intensity of the black body radiation at temperature $T$ \\
\hline & Beam wavelength \\
\hline$\left(g_{0}\right)_{Z}$ & Statistical weight of the ground state of an atom or ion with charge $Z$ \\
\hline$T_{e}$ & Electron temperature \\
\hline$(\Delta W)_{\max }$ & $\begin{array}{l}\text { Maximum interval between adjacent energy levels of atoms and ions in the } \\
\text { plasma }\end{array}$ \\
\hline$x_{\max }$ & Highest ionization energy of any of atom or ion present in the plume \\
\hline $\bar{M}$ & Average value of the plasma mass \\
\hline$r_{b}$ & The radius of the plasma boundary \\
\hline$P_{\text {tot }}$ & Total energy, supplied by the laser pulse \\
\hline$B$ & Magnetic field \\
\hline
\end{tabular}


$T_{h}$

$V_{b \infty}$

$\tau_{b}$

Temperature of the end of the laser pulse

Asymptotic expansion velocity of the plasma

Period of the plume oscillation

\section{References}

Anisimov S I, Kapeliovich B L, Perelman T L 1974 Electron emission from metal surfaces exposed to ultrashort laser pulses. Sov. Phys. JETP 39: 375-77

Anisimov S I, Lukyanchuk B S 2002 Selected problems of laser ablation theory. Phys. Uspekhi 45: 293-324

Belotserkovsky O M, Davidov Yu M 1982 Method of large particles in gas dynamics, Nauka, Moscow (in Russ.)

Capitelli M, Casavola A, Colonna G, Giacomo De A 2004 Laser-induced plasma expansion: theoretical and experimental aspects. Spectrochim. Acta Part B 59: 271-89

Casavola A, Colonna G, Capitelli M 2003 Non-equilibrium conditions during a laser-induced plasma expansion. Appl. Surf. Science 208: 85-89

Colonna G, Casavola A, Capitelli M 2001 Modeling of LIBS plasma expansion. Spectrochim. Acta Part B 56: 567-86

Drogoff Le B, Margot J, Vidal F, Laville S, Chaker M, Sabsabi M, Johnston T W, Barthelemy O 2004 Influence of the laser pulse duration on laser-produced plasma properties. Plasma Sources Sci. Technol. 13: 223-30

Furusawa H, Sakka T, Ogata Y H 2004 Characterization of ablated species in laser-induced plasma plume. J. Appl. Phys. 96: 975-82

Giacomo De A, Shakhatov V A, Pascale De O 2001 Optical emission spectroscopy and modeling of plasma produced by laser ablation of titanium oxides. Spectrochim. Acta Part B 56: 753-76

Gornushkin I B, Stevenson C L, Smith B W, Omenetto N, Winefordner J D 2001 Modeling an inhomogeneous optically thick laser-induced plasma: a simplified theoretical approach. Spectrochim. Acta Part B 56: 1769-85

Ho J R, Grigoropoulos C P, Humphrey J A C 1996 Gas dynamics and radiation heat transfer in the vapor plume produced by pulsed laser irradiation of aluminum. J. Appl. Phys. 79: 7205-15

Itina T E, Hermann J, Delaporte P, Sentis M 2003 Combined continuous microscopic modelling of laser plume expansion. Appl. Surf. Science 208: 27-32

Knight C J 1979 Theoretical modelling of rapid surface vaporization with back-pressure. AIAA Journal 17: 82-86

Kumar N 2001 Laser-induced plasma temperature. Proc. RAS 13: 3-18

Mao S S, Mao X, Grief R, Russo R E 2000 Initiation of early-stage plasma during picosecond laser ablation of solids. Appl. Phys. Lett. 77: 2464-66

Mazhukin V I, Nossov V V, Flamant G, Smirnov I 2002 Modelling of radiation transfer and emission spectra in laser-induced plasma of Al vapor. J. Quant. Spectrosc. Radiat. Transfer 73: 451-60

Noll R, Sattmann R, Sturm V, Winkelmann S 2004 Space- and time-resolved dynamics of plasmas generated by laser double pulses interacting with metallic samples. J. Anal. At. Spectrometry 19: $419-28$

Rethfeld B, Sokolwski-Tinten K, von der Linde D, Anisimov S I 2004 Timescales in the response of materials to femtosecond laser excitation. Appl. Phys. A79: 767-69

Russo R E 1995 Laser ablation. Appl. Spectroscopy 49: 14A-28A

Samarsky A A, Popov Yu M 1975 Difference schemes of gas dynamics, Nauka, Moscow (in Russ.)

Singh R K, Holland O W, Narayan J 1990 Theoretical model for deposition of superconducting thin films using pulsed laser evaporation technique. J. Appl. Phys. 68: 233-47

Stapleton M W, Mosnier J P 2002 A computational model for selected emission transitions in a laser produced lithium ablation plume. Appl. Surf. Science 197: 72-76

Stoian R, Ashkenasi D, Rosenfeld A, Campbell EEB 2000 Coulomb explosion in ultrashort pulsed laser ablation of Al2O3. Phys. Rev. B 62: 13167-73 
Stuart B C, Feit M D, Herman F, Rubenchik A M, Shore B W, Perry M D 1996 Nanosecond-tofemtosecond laser-induced breakdown in dielectrics. Phys. Rev. B53: 1749-61

Tonshoff H K, Momma C, Ostendorf A, Nolte S, Kamlage G 2000 Micro-drilling of metals with ultrashort laser pulses. J. Laser Application 12: 23-27

Vorobev V S 1993 Plasma arising during the interaction of laser radiation with solids. Phys. Uspekhi 36: $1129-57$

Zeldovich Ya B, Raizer Yu P 1967 Physics of shock waves and high temperature hydrodynamics phenomena (New York: Academic Press) 129-132 